EPJ manuscript No.

(will be inserted by the editor)

\title{
Convective and Absolute Instabilities in the Subcritical Ginzburg-Landau Equation
}

Pere Colet, Daniel Walgraef ${ }^{a}$, and Maxi San Miguel

Instituto Mediterráneo de Estudios Avanzados, IMEDEA ${ }^{\mathrm{b}}$ (CSIC-UIB), Campus Universitat Illes Balears, E-07071 Palma de Mallorca, Spain.

11 December 1998

\begin{abstract}
We study the nature of the instability of the homogeneous steady states of the subcritical Ginzburg-Landau equation in the presence of group velocity. The shift of the absolute instability threshold of the trivial steady state, induced by the destabilizing cubic nonlinearities, is confirmed by the numerical analysis of the evolution of its perturbations. It is also shown that the dynamics of these perturbations is such that finite size effects may suppress the transition from convective to absolute instability. Finally, we analyze the instability of the subcritical middle branch of steady states, and show, analytically and numerically, that this branch may be convectively unstable for sufficiently high values of the group velocity.
\end{abstract}

PACS. 47.20.Ky Fluid Dynamics: Nonlinearity - 47.54.+r Fluid Dynamics: Pattern selection; pattern formation - 05.40+j Statistical Physics and Thermodynamics: Fluctuation phenomena, random processes, and Brownian motion

\section{Introduction}

Several physico-chemical systems driven out of equilibrium present stationnary instabilities of the Turing type, or oscillatory instabilities corresponding to Hopf bifurca-

\footnotetext{
a Permanent address: Centre for Non-Linear Phenomena and Complex Systems, Université Libre de Bruxelles, Campus Plaine, Blv. du Triomphe B.P 231, B-1050 Brussels, Belgium

b Electronic address: http://www.imedea.uib.es/PhysDept/
}

tions. Such instabilities lead to the formation of various kinds of spatio-temporal patterns [1]. Well known examples are: Rayleigh-Bénard instabilities in Newtonian fluids, binary mixtures, or viscoelastic solutions [2, 3, electrohydrodynamic instabilities in nematic liquid crystals 沺, Turing instabilities in nonlinear chemical systems [5], convective instabilities in Taylor-Couette devices [6], etc. Close to such instabilities, the dynamics of the system may usu- 
ally be reduced to amplitude equations of the GinzburgLandau type, which describe the evolution of the patterns that may appear beyond the bifurcation point 7 .

According to the system under consideration, and to the nature of the instability, these Ginzburg-Landau equations may contain mean flow terms induced by group velocities. In this case, pattern formation crucially depends on the convective or absolute nature of the instability. Let us recall that, when the reference state is convectively unstable, localized perturbations are driven by the mean flow in such a way that they grow in the moving reference frame, but decay at any fixed location. On the contrary, in the absolute instability regime, localized perturbations grow at any fixed location [8]. The behavior of the system is thus qualitatively very different in both regimes. In the convectively unstable regime, a deterministic system cannot develop the expected patterns, except in particular experimental set-ups, while in a stochastic system, noise is spatially amplified and gives rise to noise-sustained structures [9,10,11]. On the contrary, in the absolutely unstable regime, patterns are intrinsically sustained by the deterministic dynamics, which provides the relevant selection and stability criteria 12, 13. Hence, the concepts of convective and absolute instability are essential to understand the behavior of nonlinear wave patterns and their stability [9, 14.

The nature of the instability of the trivial steady state has been studied, either numerically, analytically and experimentally: In the case of supercritical bifurcations, linear criteria are appropriate to determine the absolute in- stability threshold, and to analyze the transition from convective to absolute instability [9, 10, 11, 15, 16, 17, 18, 19]. However, in the case of subcritical bifurcations, the nonlinearities are destabilizing, which leads to the failure of linear instability criteria. In a qualitative analysis based on the potential character of the real subcritical GinzburgLandau equation, Chomaz [20] argued that the transition between convective and absolute instability of the trivial steady state should occur at the point where a front between the rest state and the nontrivial steady state is stationary in a frame moving with the group velocity. This defines the nonlinear convective-absolute instability threshold, above which nonlinear global modes are intrinsically sustained by the dynamics, as discussed by Couairon and Chomaz [21]. This argument relies on the existence of a unique front between the basic and the bifurcating states, as it is the case in the subcritical domain where both basic and bifurcating states are linearly stable. In the supercritical domain, where the basic state is linearly unstable, or in the complex Ginzburg-Landau equation, this front is not unique any more, and, as commented by van Hecke et al. 22], one has to know which nonlinear front solution is selected, to determine the nonlinear stability properties of the basic state.

Within this context, our aim in this paper is to contribute to the study of this problem addressing some aspects of it that so far have not been considered. Additionally we study stochastic effects. A first aspect concerns finite size effects and their influence on the transition from convective to absolute instability for the trivial 
Pere Colet et al.: Convective and Absolute Instabilities in the Subcritical Ginzburg-Landau Equation

steady state. Indeed, our numerical analysis of the evolution of perturbations of this state show that it consists in two stages. The first one is devoted to the building of a front between this state and the bifurcating one. It is during the second stage that this front moves outwards or inwards according to the convective or absolute nature of the instability. We will show that, although the absolute instability threshold may effectively be shifted, due to nonlinear effects, in agreement with [20], the first step of the evolution is sensitive to the size of the system, and this may affect the practical determination of the absolute instability threshold, and even suppress it.

A second aspect is the effect of the group velocity on the unstable subcritical branch. In the subcritical domain, there is a middle branch of steady states, between the trivial and the bifurcating ones. In fact, the nature of the instability of this branch in the presence of a group velocity has not been considered so far. In the absence of group velocity, this branch is absolutely unstable. However, the nature of the instability may be modified in systems with group velocity or mean flow effects. We will effectively show, analytically and numerically, that this unstable subcritical branch may be convectively unstable, totally or partly, according to the mean flow intensity. Effectively, in deterministic systems, unstable states on this branch do not necessarily decay in the presence of group velocity, while they may remain long lived in stochastic systems. This fact may be of practical importance, since it provides an alternative way to stabilize the subcritical middle branch, which is qualitatively different from the one proposed by Thual and Fauve [23]. It could, furthermore, provide the last building block needed for the understanding of pattern formation in binary fluid convection, as suggested in [1].

In section 2, we recall the dynamical model. In section 3 we discuss the nature of the instability of the trivial steady state, and present the results of a numerical analysis of the problem. In section \&, we show, analytically and numerically, that the subcritical middle branch of steady states may be convectively unstable, and may thus be stabilized by mean flow effects in deterministic systems. Finally, conclusions are drawn in section 5 .

\section{The Subcritical Scalar Ginzburg-Landau}

\section{Equation}

For the sake of simplicity, we will consider, in this paper, systems described by a scalar order parameterlike variable, and where the dynamics is given by the real fifth-order Ginzburg-Landau equation, which may be written, in onedimensional geometries, as 11,24 :

$$
\partial_{t} A+c \partial_{x} A=\epsilon A+\partial_{x}^{2} A+v A^{3}-A^{5}+\sqrt{\xi} \chi(x, t),
$$

For future reference we have added to the equation a stochastic term $\chi(x, t)$. This models a Gaussian white noise of zero mean and variance given by $\left\langle\chi(x, t) \chi\left(x^{\prime}, t^{\prime}\right)\right\rangle=$ $2 \delta\left(x-x^{\prime}\right) \delta\left(t-t^{\prime}\right)$. In the remainder of this section we consider the deterministic situation with $\xi=0$.

Bifurcating uniform steady states $A(x, t)=R$ of this equation are well known:

$$
R_{ \pm}^{2}=\frac{1}{2}\left(v \pm \sqrt{v^{2}+4 \epsilon}\right)
$$


The linear evolution of the perturbations $\rho_{ \pm}=A-R_{ \pm}$ around these states is then given by:

$$
\partial_{t} \rho_{ \pm}+c \partial_{x} \rho_{ \pm}=\mp 2 R_{ \pm}^{2} \sqrt{v^{2}+4 \epsilon} \rho_{ \pm}+\partial_{x}^{2} \rho_{ \pm}
$$

Hence, in the absence of group velocity, the upper branch $R_{+}$exists and is stable for $-\frac{v^{2}}{4}<\epsilon$, while the middle branch $R_{-}$exists and is unstable for $-\frac{v^{2}}{4}<\epsilon<0$ (cf. fig. 1).

This picture is, of course, known to change in the presence of a finite group velocity $c$. Let us first recall the linear and nonlinear criteria for convective and absolute instability for the trivial steady state $A=0$.

\section{Linear and Nonlinear Instability of the}

\section{Trivial Steady State}

\subsection{Analytical Results}

The linear evolution around the trivial steady state $A=0$ is given by

$$
\partial_{t} \rho_{0}+c \partial_{x} \rho_{0}=\epsilon \rho_{0}+\partial_{x}^{2} \rho_{0}
$$

and the corresponding dispersion relation is

$$
\omega=\epsilon-c \kappa+\kappa^{2}
$$

with $\kappa=k^{\prime}+i k^{\prime \prime}$. The usual linear instability criterion [8]

$$
\Re \frac{d \omega}{d \kappa}=\Im \frac{d \omega}{d \kappa}=0
$$

and $\Re(\omega(\kappa))=0$ gives that the trivial steady state is convectively unstable for $0<\epsilon<c^{2} / 4$, and absolutely unstable for $\epsilon>c^{2} / 4$.

However, since the nonlinearities of the dynamics are destabilizing, the linear terms may possibly not govern the growth of perturbations of the steady state. Hence, a reliable stability analysis has to include nonlinear terms. As discussed by Chomaz and Couairon 20,21, the nonlinear stability analysis of the trivial steady state relies on its response to perturbations of finite extent and amplitude. Hence, in the case eq. (1), without group velocity $(c=0)$, it is sufficient to consider a front solution joining the 0 state at $x \rightarrow-\infty$ to the $R_{+}$state at $x \rightarrow+\infty$.

In the case of the dynamics given by Eq. (11), this velocity, $c_{f}$ may be calculated exactly [25], and is found to be (cf. fig. 2)

$$
\begin{aligned}
& c_{f}=c^{\dagger}=\frac{1}{\sqrt{3}}\left(-v+2 \sqrt{v^{2}+4 \epsilon}\right)\left(\text { for }-\frac{v^{2}}{4}<\epsilon<\frac{3 v^{2}}{4}\right) \\
& c_{f}=c^{*}=2 \sqrt{\epsilon}\left(\text { for } \frac{3 v^{2}}{4}<\epsilon\right)
\end{aligned}
$$

Note that $c^{*}$ is the linear marginal velocity.

If the front velocity is negative, which is the case for $\epsilon<-3 v^{2} / 16$, an isolated droplet of the $R_{+}$state embedded into the 0 state shrinks, and the 0 state is stable. On the contrary, if $c_{f}$ is positive, which is the case for $\epsilon>-3 v^{2} / 16, R_{+}$droplets grow, and the 0 state is nonlinearly unstable. The value $\epsilon=-3 v^{2} / 16$ corresponds to the Maxwell construction of phase transitions in which the trivial and upper branch have equal stability.

When $c \neq 0$ and $v=1$, Chomaz [20] showed that, in the unstable domain $\left(\epsilon>-3 v^{2} / 16\right)$, the instability is nonlinearly convective (NLC) when $c_{f}<c$, since, in this case, although expanding, a $R_{+}$droplet is finally advected out of the system. On the contrary, when $c_{f}>c$, the instability is absolute (NLA), since, in this case, $R_{+}$droplets expand in such a way that they finally invade the system. 
Pere Colet et al.: Convective and Absolute Instabilities in the Subcritical Ginzburg-Landau Equation

Hence, on generalizing this argument to arbitrary valued of $v$, one obtains imposing $c_{f}=c$ in Eq. (7) that the transition from convective to absolute instability occurs at:

$$
\begin{aligned}
\epsilon_{a} & =\frac{3}{16}\left(c^{2}+\frac{2}{\sqrt{3}} v c-v^{2}\right)(\text { for } c<\sqrt{3} v) \\
& =\frac{1}{4} c^{2}(\text { for } c>\sqrt{3} v)
\end{aligned}
$$

From this result, it appears clearly that, when group velocity effects dominate over nonlinear ones $(c>\sqrt{3} v)$, the absolute instability threshold remains the linear one. However, when nonlinearities dominate $\left(v>\frac{c}{\sqrt{3}}\right)$, the absolute instability threshold decreases, but remains in the $\epsilon>0$ domain, when $v<c \sqrt{3}$. It only becomes negative when $v>c \sqrt{3}$. This last case is the one originally considered in [20.

\subsection{Numerical Analysis}

The above results have been checked through the numerical integration of the equation (11). We will present here some of the data obtained for systems being initially in the trivial steady state, and compare them to the predictions obtained from the analytical analysis outlined in the preceeding section. To observe a convective instability we consider a semi-infinite system with one of the boundaries anchored to the unstable state $A(x=0)=0$. Experimentally, this boundary condition can be achieved using a negative value for the control parameter $\epsilon$ for $x<0$.

The numerical integrations have been performed using a finite difference method [19] with a spatial step of $\delta x=$ 0.05 and time step $\delta t=0.001$, except where otherwise noted. As explained before, the boundary conditions for a system of size $L$ were taken as follows: $A=0$ at $x=0$ for all times and $\partial_{x} A=0$ at $x=L$.

We only discuss here situations where the nonlinearities dominate over mean flow effects, thus where linear instability criterion fails.

(1) A first case corresponds to $c=v=1$. In this case, the transition from convective to absolute instability should occur at $\epsilon_{a}=\frac{\sqrt{3}}{8} \simeq 0.21$. This is illustrated by the numerical results presented in fig. 3. In fig. 3 (a) and (b), we show the deterministic evolution of the field $A$ from random initial conditions around $A=0$ and for $\epsilon=0.18$. The data confirm the convective nature of the instability. Effectively, we see, in a first stage, the building of a front between the trivial state and the bifurcating one, and, in a second stage, this front is advected out of the system. On the contrary, for $\epsilon=0.23$, the instability is absolute, as shown in fig. 3 (c) and (d), where the front moves in the opposite direction, and the bifurcating state invades the system.

The difference between subcritical and supercritical behavior is enlightened in fig. 3 (e) and (f), where the field evolution has been computed with the same parameters as in fig. 3 (c) and (d), except that $v$ has been changed from +1 to -1 to simulate supercriticality. In this case, the instability should be convective, since the absolute threshold is $\epsilon=0.25$, and the results are in agreement with this prediction.

The effect of noise in the regime of convective instability is presented in fig. 4. The field dynamics has been 
computed for the same values of the parameters as in fig.

3 (a), but in the presence of noise of different intensities. The noise intensity has been fixed at $\xi=10^{-6}$ in fig. (1) and at $\xi=10^{-14}$ in fig. 1 (b). In both cases we observe noise sustained structures: Noise is able to sustain finite field amplitudes (positive or negative, according to the,+- symmetry of the system). Weaker noise induces larger healing length for the pattern. Hence, in the stochastic case, pattern formation is sensitive to system size, since the latter has to be larger than the healing length, for the pattern to be able to develop.

(2) In a second case, we chose $c=0.5, v=1.5$, and this corresponds to the situation presented by Chomaz [20], where nonlinear effects dominate $(v>c \sqrt{3}$. $)$ and where the transition from convective to absolute instability occurs in the subcritical domain since $\epsilon_{a} \simeq-0.21$. For $\epsilon_{a}<\epsilon$, the instability is absolute, but the dynamics is qualitatively different if $\epsilon$ is positive or negative. When $\epsilon>0$, both linear and cubic terms are destabilizing, and the building and propagation of fronts between trivial and bifurcating states is much faster than for $\epsilon<0$, when the linear term is stabilizing, and the cubic one is destabilizing. When the dynamics becomes very slow the time and system size needed to see the formation of a front from an initial perturbation become very large, so that even in the absolutely unstable regime one might not observe the decay of the state $A=0$ in finite times for a finite system. This effect is illustrated in fig. 5 which corresponds to the absolutely unstable regime. Note the significant increase of the times scales in comparison with figs. 3 and 4 , de- spite the fact that the perturbation of the zero state at the initial time is much large (see the figure caption). We note that for $\epsilon<0$ the evolution would still be slower. We first observe the formation of the front ( initially moving to the right) and much later, when it reaches the upper branch, invading the whole syste. Hence, when the characteristic length needed for the building of the front is larger than the system size, the instability is effectively convective, although the system should be in the absolute instability regime (in the sense of semi-infinite geometries). For the parameters chosen in this example and for a length $L<2000$ one does not observe the decay of the state $A=0$.

It is worthnoting that the observed finite size effects confirm and complement the analysis made by Chomaz and Couairon 26 of fully nonlinear solutions of GinzburgLandau equations in finite domains. In case (1), for $\epsilon=$ 0.23, nonlinear global (NLG) modes exist, even in finite domains. However, since the basic state, $A=0$ is linearly absolutely stable, NLG modes only develop if the initial condition is sufficiently large for the transients to reach an order one amplitude in the finite domain. Since the amplification factor increases exponentially with $L$, the minimum amplitude of initial perturbations able to trigger the NLG mode decreases exponentially with $L$ [26]. As a result, the development of NLG modes is almost insensitive, in most practical situations, to system size.

On the contrary, in case (2), the basic state is linearly stable, absolutely and convectively, and the minimum amplitude of initial perturbations able to trigger NLG modes 
Pere Colet et al.: Convective and Absolute Instabilities in the Subcritical Ginzburg-Landau Equation

in finite boxes decreases linearly with $L$. It is why, in the conditions of our numerical analysis, no global mode is obtained for $L<2000$.

\section{Stability Analysis of the Bifurcating States}

\subsection{Analytical Results}

The linear evolution around the upper branch steady states $R_{+}$and middle branch steady states $R_{-}$is given by eq. (3). The upper states $R_{+}$are linearly stable for all $\epsilon>-\frac{v^{2}}{4}$.

On the other hand, the usual linear instability criterion shows that the $R_{-}$steady states are convectively unstable for $8 R_{-}^{2} \sqrt{v^{2}+4 \epsilon}<c^{2}$, and absolutely unstable for $8 R_{-}^{2} \sqrt{v^{2}+4 \epsilon}>c^{2}$. In other words, these states are absolutely unstable in the range

$-\frac{1}{8}\left(v^{2}+\frac{c^{2}}{2}+v \sqrt{v^{2}-c^{2}}\right)<\epsilon<-\frac{1}{8}\left(v^{2}+\frac{c^{2}}{2}-v \sqrt{v^{2}-c^{2}}\right)$

and convectively unstable in the windows defined by

$$
-\frac{v^{2}}{4}<\epsilon<-\frac{1}{8}\left(v^{2}+\frac{c^{2}}{2}+v \sqrt{v^{2}-c^{2}}\right)
$$

and

$$
-\frac{1}{8}\left(v^{2}+\frac{c^{2}}{2}-v \sqrt{v^{2}-c^{2}}\right)<\epsilon<0
$$

Hence, when $v^{2}<c^{2}$, these steady states are always linearly convectively unstable. Still, when $v^{2}>c^{2}$, there is a range of linear absolute instability in the middle of their domain of existence, and a range of linear convective instability close to the points where these states disappear. This is shown in fig. 6 .

Nevertheless the linear stability criteria may fail in the presence of destabilizing nonlinearities. This is not only the case for the evolution of the perturbations of the trivial steady state since the bifurcation is subcritical, but it may also be the case for the perturbations around the middle steady state branch, whose evolution is given by

$$
\begin{aligned}
\partial_{t} \rho_{-} & +c \partial_{x} \rho_{-}=+2 R_{-}^{2} \sqrt{v^{2}+4 \epsilon} \rho_{-}+\partial_{x}^{2} \rho_{-} \\
& -R_{-}\left(2 v-5 \sqrt{v^{2}+4 \epsilon}\right) \rho_{-}^{2}-\left(4 v-5 \sqrt{v^{2}+4 \epsilon}\right) \rho_{-}^{3} \\
& -5 R_{-} \rho_{-}^{4}-\rho_{-}^{5}
\end{aligned}
$$

The quadratic nonlinearity is destabilizing for $\epsilon<\epsilon_{L}=$ $-0.21 v^{2}$. In such cases, one has to perform a nonlinear analysis of the dynamics to determine the convective or absolute nature of the instability.

In the regime where the nonlinearities of the evolution equation (12) are stabilizing, i.e. for $\epsilon_{L}=-0.21 v^{2}<\epsilon<$ 0, the results of the linear analysis may be assumed to be valid. Hence, we may safely rely on these results above the metastability point, i.e. for $\epsilon_{M}=-3 / 16 v^{2}<\epsilon<0$. Below the metastability point, i.e. for $-0.25 v^{2}<\epsilon<$ $-3 / 16 v^{2}$, one has to perform a nonlinear analysis, which, in this case, relies on the evolution of fronts between middle branch states and the trivial steady state. We do not perform this analysis here since it would only affect quantitatively but not qualitatively the results presented above.

\subsection{Dynamics of the Subcritical Unstable Branch}

We have numerically confirmed the convective nature of the instability of the subcritical middle branch. The numerical integration has been performed as indicated in subsection 3.2. Also, as indicated in that subsection, to observe a convective instability we consider a semi-infinite 
system with one of the boundaries anchored to the unstable state. Here we have to take $A(x=0)=R_{-}$corresponding to the field amplitude of the subcritical middle branch. Experimentally, this boundary condition can not be achieved as easily as before because there is no value of the control parameter $\epsilon$ for which $A(x)=R_{-}$is an homogeneous steady stable state. However depending on the system it can be imposed in different ways. In an optical system, for example, the left boundary condition could be achieved injecting an external field at $x=0$ with the apropriate amplitude. Finally, as in subsection 3.2, the right boundary condition is taken as $\partial_{x} A=0$ at $x=L$

We computed the evolution from an initial steady state with $R_{-}^{2}=0.1$ on the middle branch, which corresponds to $v=1$ and $\epsilon=-0.09$. We then study the system dynamics for different values of the group velocity $c$. According to the previous discussion, for

(1) $c<0.8$, the state $R_{-}$should be absolutely unstable

(2) $c>0.8$, the state $R_{-}$should be convectively unstable.

In fig. 17 (a), we present, for $c=1$, the results obtained for the deterministic evolution of an initial perturbation of the state $R_{-}$. They show that the instability is effectively convective. On lowering the group velocity from $c=1$ to $c=0.55$, the nature of the instability changes from convective to absolute, as expected, and shown in fig. 7 (b). These results confirm that the middle branch, which is always stable for $c=0$, may be stabilized by mean flow effects in deterministic systems, in the sense that there is a range of parameters in which it is only convectively unstable.

The effect of noise in the convectively unstable regime of the trivial state was to sustain a structure continuously excited by noise. In the case of the middle branch. $R_{-}$, and when this is convectively unstable, noise forces the system to relax randomly to either of the two coexisting stable branches, as shown in fig. 8. Still, if noise is weak in comparison with the strength needed to see its effect in a finite system, one would observe the middle branch as effectively stable.

\section{Conclusions}

In this paper, we considered systems described by the subcritical Ginzburg-Landau equation, and analyzed some problems related with the effect of group velocities on the stability of its steady states. In the case of the trivial steady state, it is known that the transition between convective and absolute linear instability regimes is shifted by the effect of destabilizing nonlinearities, and the corresponding nonlinear absolute instability threshold may easily be computed for semi-infinite systems [20,21]. Our numerical study of the evolution of perturbations from the trivial steady state in finite systems shows that, in a first step, a front is built between this state and the bifurcating one, which corresponds to the upper branch of steady states. Then, according to the intensity of the group velocity, the front moves outwards or inwards, which corresponds to convective or absolute instability, respectively. When the characteristic length needed for the building of 
the front is shorter than the system size, the nature of the instability is in agreement with the theoretical predictions made for semi-infinite systems. However, our numerical results show that, if the characteristic building length of the front is larger than the system size, one will never see inward motion of the front, and, in this case, even above the absolute instability threshold, the instability is effectively convective.

We also studied the instability of the subcritical middle branch of steady states, a problem that had not been addressed up to now. It may be shown, already at the level of a linear analysis, that this branch, which is absolutely unstable without group velocity, may entirely become convectively unstable in the presence of group velocities larger than some well-defined critical value. This result has been confirmed by the numerical analysis of the evolution of perturbations of steady states on this branch. The stabilization of such steady states has effectively be obtained, in deterministic systems, for group velocities in the predicted range. In stochastic systems, however, these steady states relax to one of the stable branches, as expected. Nevertheless, for this relaxation to occur, either noise strength or system size have to be large enough. This effect may be of practical importance, for example, in binary fluid convection, where, besides the fact that the role of subcriticality is not clearly understood yet 27, the presence of natural or forced mean flows, or group velocities, could effectively stabilize otherwise unstable branches of steady states.

\section{Acknowledgements}

Financial support from DGICYT (Spain) Project PB941167 is acknowledged. DW is supported by the Belgian National Fund for Scientific Research. The authors also acknowledge helpful discussions with W. van Saarloos.

\section{References}

1. M.C. Cross and P.C. Hohenberg, Rev. Mod. Phys. 65, 851 (1993).

2. R. Walden, P. Kolodner, A. Passner and C.M. Surko, Phys. Rev. Lett. 55, 496 (1985).

3. R.G. Larson, Rheol. Acta 31, 213-263 (1992).

4. A. Joets and R. Ribotta in Propagation in Systems far from Equilibrium, J.E.Wesfreid et al. eds., Springer, New York, p.176 (1988).

5. P. Borckmans, G. Dewel, A. De Wit and D. Walgraef, in Chemical Waves and Patterns, R. Kapral and K. Showalter eds., Kluwer, Dordrecht, pp.323-363,(1994).

6. C.D. Andereck and F. Hayot, Ordered and Turbulent Patterns in Taylor-Couette Flow, Plenum, New York, 1992.

7. D. Walgraef, Spatio-Temporal Pattern Formation. with examples in Physics, Chemistry ans Materials Science, Springer-Verlag, New York, 1996.

8. P. Huerre in Instabilities and Nonequilibrium Structures, E. Tirapegui and D. Villarroel eds., Reidel, Dordrecht, (1987), 141.

9. R.J. Deissler, J. Stat. Phys., 40, 376 (1985); 54, 1459 (1989). R.J. Deissler, Physica D 25, 233 (1987).

10. K.L. Babcock, G. Ahlers, D.S. Cannell, Phys. Rev. E 50, 3670, (1994). 
11. M. Santagiustina, P. Colet, M. San Miguel, D. Walgraef, Phys. Rev. Lett. 79, 3633 (1997).

12. H.W. Mueller, M. Luecke and M. Kamps, Phys. Rev. A 45, 3714 (1992).

13. P. Buechel, M. Luecke, D. Roth and R. Schmitz, Phys. Rev. E 53, 4764 (1996).

14. H.W. Mueller and M. Tveitereid, Phys. Rev. Lett. 74, 1582 (1995).

15. R.J. Deissler and H.R. Brand, Phys. Lett. A 130, 293 (1988).

16. H.R. Brand, R.J. Deissler and G. Ahlers, Phys. Rev. A 43, $4262(1991)$

17. A. Tsameret and V. Steinberg, Europhys. Lett. 14, 331, (1991); Phys. Rev. E 49, 1291, (1994).

18. M. Luecke and A. Recktenwald, Europhys. Lett. 22, 559 (1993).

19. M. Neufeld, D. Walgraef and M. San Miguel, Phys. Rev. E 54, 6344 (1996).

20. J.M. Chomaz, Phys. Rev. Lett. 69, 1931 (1992).

21. A. Couairon and J.M. Chomaz, Phys. Rev. Lett. 77,4015 (1996); Physica D 108, 236 (1997).

22. M. van Hecke, W. van Saarloos and P.C. Hohenberg, Phys. Rev.Lett. 71, 2162 (1993).

23. O. Thual and S. Fauve, J. Physique (Paris) 49, 1829, (1988).

24. W. van Saarloos and P.C. Hohenberg, Physica D 56, 303 (1992).

25. W. van Saarloos, Phys. Rev. A 39, 6367 (1989).

26. J.M.Chomaz and A.Couairon, Against the Wind, preprint, LadHyX, Palaiseau, France, 1998.

27. P. Kolodner, S. Sliman, N. Aubry and R. Lima, Physica D 85, 165 (1995).

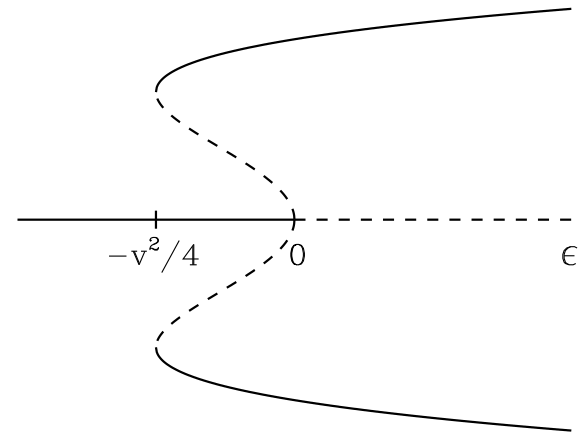

Fig. 1. Bifurcation diagram for the real subcritical scalar Ginzburg-Landau equation.

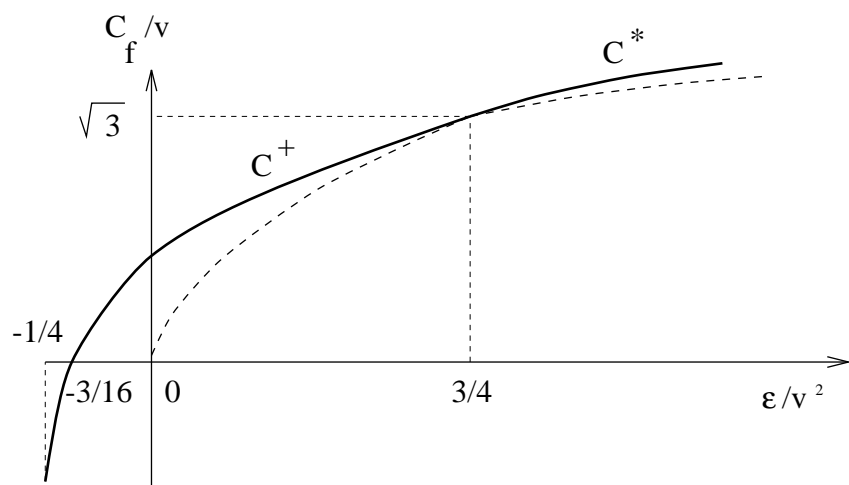

Fig. 2. Representation of the linear and nonlinear front velocities $\left(c^{*}\right.$ and $\left.c^{\dagger}\right)$ in the $\left(c_{f} / v, \epsilon / v^{2}\right)$ plane. The solid line represents the selected front velocity. The dashed lines represent $c^{*}$ for $\epsilon<3 v^{2} / 4$ and $c^{\dagger}$ for $\epsilon>3 v^{2} / 4$ 

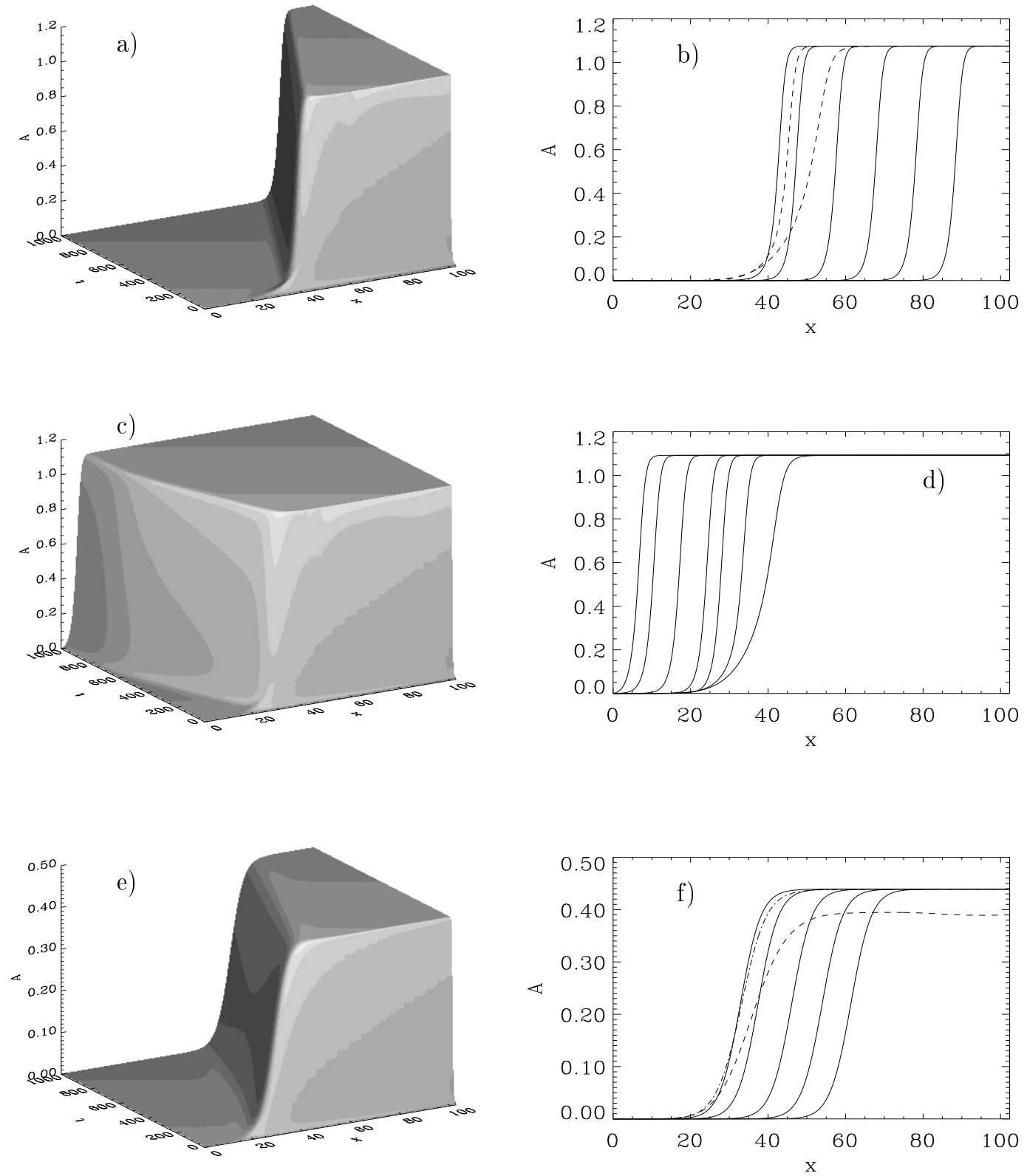

Fig. 3. Deterministic evolution of perturbations of the trivial steady state. The column on the left shows the spatio-temporal evolution of the field $A$ and the column on the right the value of the field at different times. The initial condition for each grid point is $A_{i}(t=0)=f\left|\eta_{i}\right|$, where $\eta$ is a Gaussian random number of zero mean and variance 1 and $f=10^{-4}$. In (a) and (b) we consider the subcritical case in a convectively unstable regime, with $\epsilon=0.18, v=1, c=1$. In (c) and (d) we consider the subcritical case in an absolutely unstable regime, with $\epsilon=0.23, v=1, c=1$. In (e) and (f) we consider the supercritical case, with $\epsilon=0.23, v=-1, c=1$. Dashed lines in (b) and (f) correspond to early times when the front is being formed and it effectively moves to the left. Continuous lines show the front moving to the right in (b) and (f) and moving to the left in (d) 

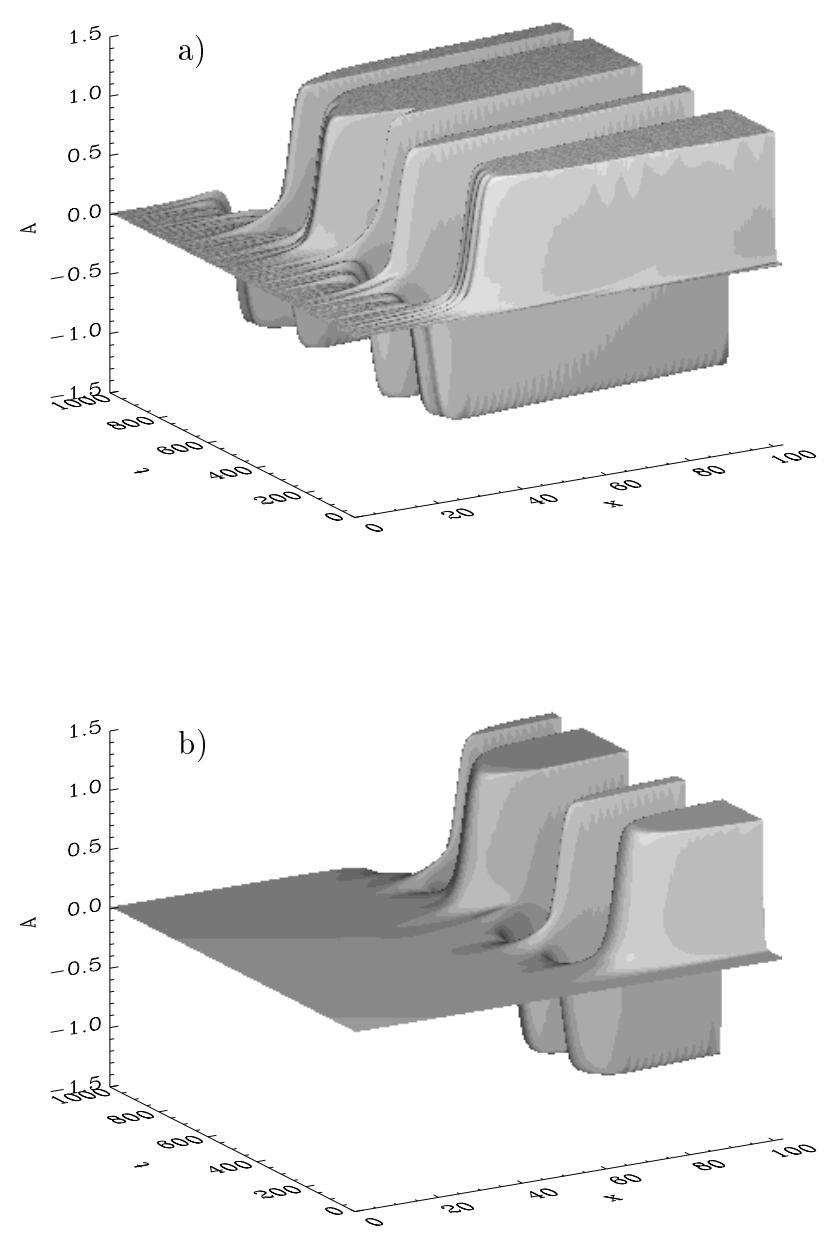

Fig. 4. Spatio-temporal evolution of the field $A$ with noise in a convectively unstable regime for $\epsilon=0.18, v=1, c=1$.

The initial condition is $A(x, t=0)=0$. (a) noise intensity $\xi=10^{-6}$, (b) noise intensity $\xi=10^{-14}$.

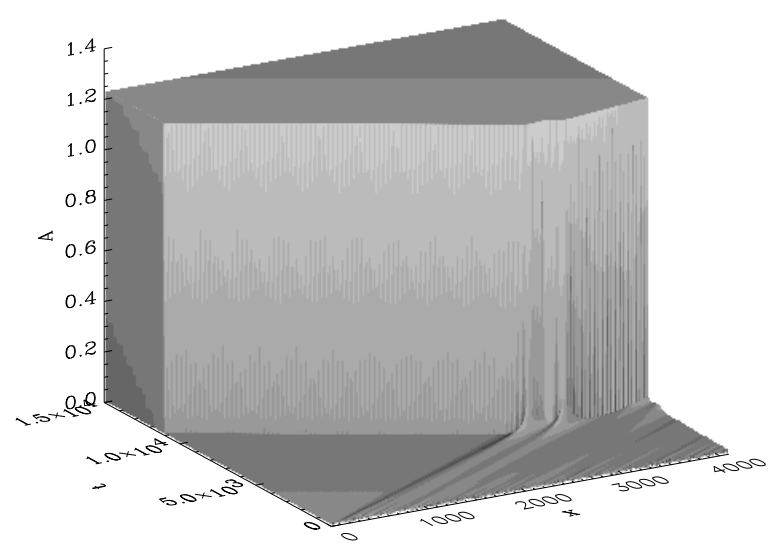

Fig. 5. Spatio-temporal evolution of the field $A$ without noise in an absolutely unstable regime with $\epsilon=0, v=1.5, c=0.5$. The initial condition is as in Fig. 3 but with $f=10^{-2}$. In this case we have taken $\delta x=1, \delta t=0.2$.

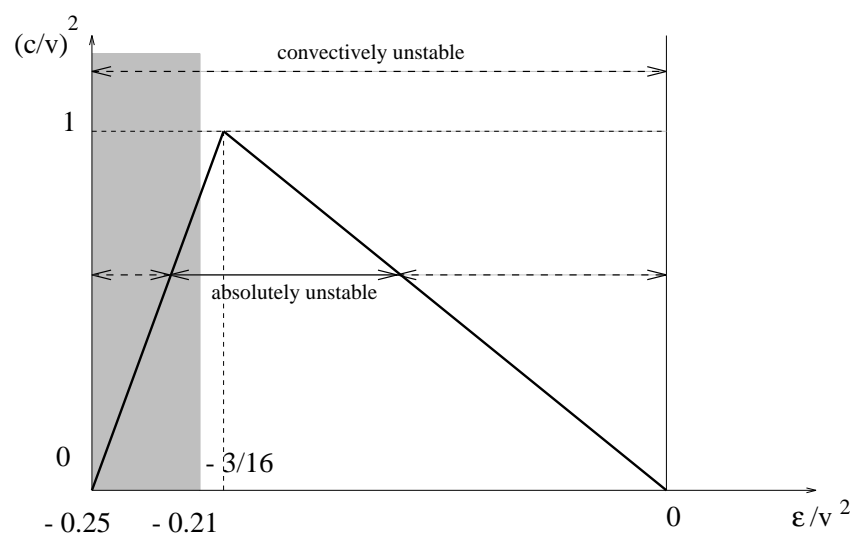

Fig. 6. Domains of linear convective and absolute instability of the middle branch uniform steady state in the $\left((c / v)^{2}, \epsilon / v^{2}\right)$ plane. The linear stability analysis is not valid in the hatched domain where the nonlinearities are destabilizing. 
Pere Colet et al.: Convective and Absolute Instabilities in the Subcritical Ginzburg-Landau Equation
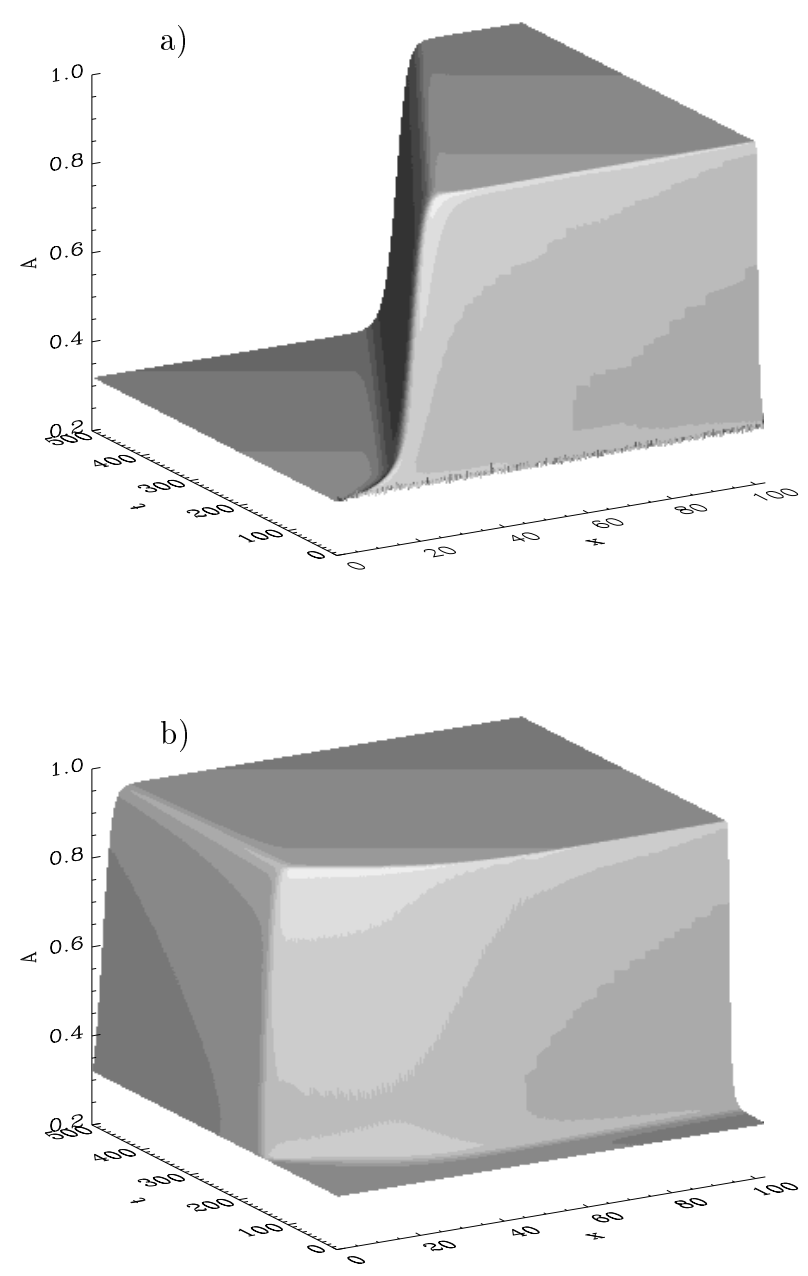

Fig. 7. Deterministic evolution of a perturbation around the middle branch state $R_{-}$in a convectively unstable regime for $\epsilon=-0.09, v=1$. The initial condition for each grid point is $A_{i}(t=0)=R_{-}+f\left|\eta_{i}\right|$, where $\eta$ is a Gaussian random number of zero mean and variance 1 and $R_{-}=\sqrt{0.1}$. (a) convectively unstable regime with $c=1$ and $f=10^{-2}$. (b) absolutely unstable regime with $c=0.55$ and $f=10^{-6}$.

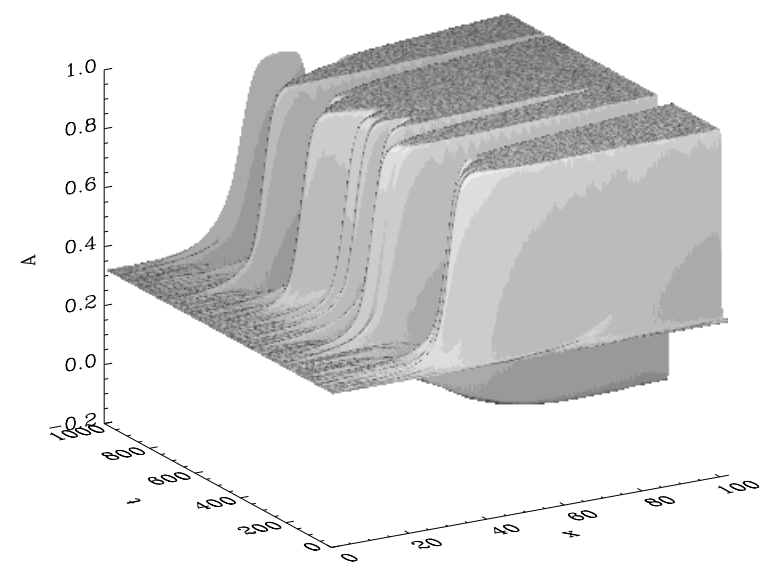

Fig. 8. Spatio-temporal evolution of the field $A$ with noise in a convectively unstable regime for the middle branch for $\epsilon=-0.09, v=1, c=1$ and $\xi=10^{-6}$. The initial condition is $A(x, 0)=\sqrt{0.1}$. 